\title{
Minimum-Norm Estimation for Binormal Receiver Operating Characteristic (ROC) Curves
}

\author{
Ori Davidov and Yuval Nov* \\ Department of Statistics, University of Haifa, Mount Carmel 31905, Israel \\ Received 28 May 2009, revised 27 July 2009, accepted 2 September 2009
}

\begin{abstract}
The receiver operating characteristic (ROC) curve is often used to assess the usefulness of a diagnostic test. We present a new method to estimate the parameters of a popular semi-parametric ROC model, called the binormal model. Our method is based on minimization of the functional distance between two estimators of an unknown transformation postulated by the model, and has a simple, closed-form solution. We study the asymptotics of our estimators, show via simulation that they compare favorably with existing estimators, and illustrate how covariates may be incorporated into the norm minimization framework.
\end{abstract}

Key words: Binormal model; Diagnostic test; Norm minimization; ROC curve; Semi-parametric inference.

Supporting Information for this article is available from the author or on the WWW under http://dx.doi.org/10.1002/bimj.200900128.

\section{Introduction}

A diagnostic test can discriminate between diseased and healthy subjects when its result is distributed differently in the two populations. Without loss of generality, the test result may be assumed to be typically higher for diseased subjects, so subjects whose result is greater than some threshold $c$ are classified as diseased, and otherwise as healthy.

Let $X$ and $Y$ denote the test results of a healthy and a diseased subjects, respectively. Both are assumed to be real numbers on a continuous scale. For a fixed $c$, the sensitivity of the test is $\operatorname{Se}(c)=P(Y>c)$, which is the probability of classifying correctly a diseased subject; the specificity of the test is $\operatorname{Sp}(c)=P(X \leq c)$, which is the probability of classifying correctly a healthy subject. An ideal test would enjoy both high sensitivity and high specificity, but apart from trivial cases, these two requirements are impossible to satisfy simultaneously, as higher sensitivity, due to a low $c$, means lower specificity, and vice versa.

The receiver operating characteristic (ROC) curve is a widely used statistical device for evaluating the accuracy of a diagnostic test, as it captures the trade-off between sensitivity and specificity across all possible choices of $c$. The curve is the plot of the pairs $(1-\operatorname{Sp}(c), \operatorname{Se}(c))$ as $c$ varies from $-\infty$ to $\infty$, and can alternatively be viewed as the graph of the function $\operatorname{ROC}(t)=1-G\left(F^{-1}(1-t)\right)$ for $t \in[0,1]$, where $F$ and $G$ are the cumulative distribution functions of $X$ and $Y$, respectively. Of special interest is the area under the ROC curve, defined as AUC $=\int_{0}^{1} \mathrm{ROC}(t) \mathrm{d} t$, as it summarizes in a single number the abovementioned trade-off.

\footnotetext{
* Correspondence author: e-mail: yuval@stat.haifa.ac.il, Phone: +972-4-824-0203, Fax: +972-4-824-0204
} 
We focus in this work on a popular semi-parametric ROC model, known as the binormal model, according to which there exists an unknown, strictly increasing transformation $h: \mathbb{R} \rightarrow \mathbb{R}$ such that both $h(X)$ and $h(Y)$ are normally distributed. Since the ROC curve is invariant under increasing monotonic transformations of the data, we may assume without loss of generality that $h(X) \sim$ $N(0,1)$ and $h(Y) \sim N\left(\mu, \sigma^{2}\right)$, where $\mu>0$ and $\sigma>0$ are unknown parameters to be estimated. Under the binormal model, $\operatorname{ROC}(t)=\Phi\left(\sigma^{-1}\left(\mu+\Phi^{-1}(t)\right)\right), 0 \leq t \leq 1$, and

$$
\mathrm{AUC}=\Phi\left(\frac{\mu}{\sqrt{1+\sigma^{2}}}\right)
$$

where $\Phi$ is the cumulative distribution function of the standard normal distribution

For a comprehensive review of the general ROC literature, see the books of Zhou, Obuchowski, and McClish (2002) and Pepe (2003). Early work on the binormal ROC model dealt with ordinal, rather than continuous, data (Dorfman and Alf, 1968; Ogilvie and Creelman, 1968). Metz, Herman, and Shen (1998) showed how, by grouping truth-state runs of the data, a continuous binormal ROC estimation problem can be reduced to an ordinal one, and developed a maximum-likelihood and a quasi-maximum-likelihood algorithms for ROC curve fitting. Hsieh and Turnbull (1996) noted that in the binormal setting, the ordinal dominance curve (a curve closely related to the ROC curve) has a rather simple parametric form: $F\left(G^{-1}(t)\right)=\Phi\left(\mu+\sigma \Phi^{-1}(t)\right)$. They provide two methods for estimating $\mu$ and $\sigma$ that capitalize on the last equality - one for grouped data, and another, which is more closely related to ours, for continuous ungrouped data. (We discuss the differences between our work and previous methods in Section 6, after we present our approach.) Pepe (2000) and Alonzo and Pepe (2002) take the parametric, distribution-free (PDF) approach to ROC analysis, and model the ROC curve directly, without addressing the underlying distributions or the transformation $h$; the binormal ROC curve is a special case of their more general formulation, and they derive their estimators through binary indicator regression. Following the PDF approach, Zhang and Pepe (2005) focused on comparing ROC curves of two markers of a disease, and on incorporating categorical covariates. Zou and Hall (2000) used Monte Carlo and other numerical methods to solve the score equation of the ranking data under the binormal assumption, and thus estimate the model parameters. Cai and Moskowitz (2004) derived semi-parametric efficient maximum-profile-likelihood estimators and, in addition, developed a pseudo-maximum-likelihood estimation procedure that allows for covariates to be incorporated into the model. Zhou and Lin (2008) proposed a semi-parametric maximum-likelihood approach for inference under the binormal ROC models, which yields also an estimate of the unknown transformation $h$; they presented a two-stage iterative procedure to solve the corresponding maximization problem. Gu and Ghosal (2009) presented a Bayesian estimation approach, which uses rank-based likelihood. Hanley (1996) reported that binormal inference is robust, in the sense that various other bi-distributional forms, when rescaled properly (without changing the ROC curve or the area below it), resemble closely the binormal model. Faraggi and Reiser (2002) compared via simulation various AUC estimation procedures, both from models that relax the binormality assumption and from models that impose further restrictions on it, and studied the robustness of these procedures.

In this work we present a new approach for estimating ROC curves under the binormal assumption. The approach, which is based on norm minimization, is intuitively easy to understand, simple to implement, and provides an immediate estimator for the unknown function $h$. We present the method in Section 2, and study its asymptotics in Section 3. Section 4 is a numerical study, in which we present graphically simulated estimates of $h$, compare, via simulation, the performance of our estimators with that of other estimators, and analyze a clinical data set. We outline how covariates may be incorporated into our model and analysis in Section 5, and conclude in Section 6 with a discussion. 


\section{Minimum-norm Estimation}

The central idea of this paper is to estimate the unknown function $h$ in two different ways; only one of the two estimates depends on the parameters $\mu$ and $\sigma$, and we estimate $\mu$ and $\sigma$ to be the values that make the two estimates of $h$ as similar as possible, that is, the values that minimize the difference between them, as measured by a certain norm.

Let $X_{1}, \ldots, X_{m}$ be i.i.d. random variables having cumulative distribution function $F$, corresponding to test results from $m$ healthy subjects, and let $Y_{1}, \ldots, Y_{n}$ be i.i.d. random variables having cumulative distribution function $G$, corresponding to test results from $n$ diseased subjects. Both the $X_{i}$ 's and the $Y_{i}$ 's are assumed to be continuous random variables, and to satisfy the binormal assumption, as stated in the introduction.

By the probability integral transform theorem, $F\left(X_{i}\right) \sim U(0,1)$; from the theorem's "converse," we obtain $\Phi^{-1}\left(F\left(X_{i}\right)\right) \sim N(0,1)$, that is, $\Phi^{-1}\left(F\left(X_{i}\right)\right)$ has the same distribution as $h\left(X_{i}\right)$. In a similar fashion, we get that $\Phi_{\mu, \sigma}^{-1}\left(G\left(Y_{i}\right)\right) \sim N\left(\mu, \sigma^{2}\right)$ (where $\Phi_{\mu, \sigma}^{-1}$ denotes the inverse of the $N\left(\mu, \sigma^{2}\right)$ cumulative distribution function), so that $\Phi_{\mu, \sigma}^{-1}\left(G\left(Y_{i}\right)\right)$ and $h\left(Y_{i}\right)$ have the same distribution. Since both $\Phi^{-1} \circ F$ and $\Phi_{\mu, \sigma}^{-1} \circ G$ are continuous and strictly increasing on the supports of the $X_{i}$ 's and the $Y_{i}$ 's, respectively, it follows from Lemma A.1 in the Appendix (applied first to $W=X_{i}, h_{1}=h$, and $h_{2}=\Phi^{-1} \circ F$, and then to $W=Y_{i}, h_{1}=h$, and $\left.h_{2}=\Phi_{\mu, \sigma}^{-1} \circ G\right)$ that

$$
h=\Phi^{-1} \circ F \quad \text { and } \quad h=\Phi_{\mu, \sigma}^{-1} \circ G .
$$

Let $\widehat{F}$ be the empirical distribution function of the $X_{i}^{\prime}$ s, and let $\widehat{G}$ be that of the $Y_{i}^{\prime}$ 's. The first equality in (2) suggests that one could estimate $h$ through $\Phi^{-1} \circ \widehat{F}$; the second suggests that if $\mu$ and $\sigma$ were known, then $\Phi_{\mu, \sigma}^{-1} \circ \widehat{G}$ would be another estimator of $h$. The main idea is to estimate $\mu$ and $\sigma$ through the values that minimize the functional distance between the two estimators just mentioned, that is,

$$
(\widehat{\mu}, \widehat{\sigma})=\underset{\mu, \sigma}{\operatorname{argmin}}\left\|\Phi^{-1} \circ \widehat{F}-\Phi_{\mu, \sigma}^{-1} \circ \widehat{G}\right\|=\underset{\mu, \sigma}{\operatorname{argmin}}\left\|\Phi^{-1} \circ \widehat{F}-\left(\mu+\sigma \Phi^{-1} \circ \widehat{G}\right)\right\|,
$$

for some norm $\|\cdot\|$. The second equality follows from the identity $\Phi_{\mu, \sigma}^{-1}=\mu+\sigma \Phi^{-1}$, which is used several times below.

We consider in this work $L_{p}[\underline{u}, \bar{u}]$ norms, for some $p \geq 1$ and $\underline{u}<\bar{u}$; that is, $\|f\|=\left(\int_{u}^{\bar{u}}|f(u)|^{p} \mathrm{~d} u\right)^{1 / p}$ for $1 \leq p<\infty$, and $\|f\|=\operatorname{ess~sup}_{\underline{u} \leq u \leq \bar{u}}|f(u)|$ for $p=\infty$ (where ess sup is the essential supremum; see Royden, 1988). Regardless of the choice of $p$ for the norm, $\underline{u}$ should not be too small and $\bar{u}$ not too large: if $u \geq \max X_{i}$, for example, then $\widehat{F}(u)=1$, and hence $\Phi^{-1}(\widehat{F}(u))=\infty$, and the norm in $(3)$ is not well defined. We discuss in Section 2.3 possible choices of $\underline{u}$ and $\bar{u}$.

\section{1 $L_{2}$-norm minimization}

The $L_{2}$-norm is the most tractable among $L_{p}$-norms for our problem. Under the $L_{2}$-norm, the optimization problem (3) becomes

$$
\underset{\mu, \sigma}{\operatorname{minimize}} \int_{\underline{u}}^{\bar{u}}\left[\Phi^{-1}(\widehat{F}(u))-\left(\mu+\sigma \Phi^{-1}(\widehat{G}(u))\right)\right]^{2} \mathrm{~d} u,
$$

which is convex and quadratic in $\mu$ and $\sigma$, with closed-form solution

$$
\widehat{\mu}=\widehat{S}_{1}-\widehat{\sigma} \widehat{S}_{2}, \quad \widehat{\sigma}=\frac{\widehat{S}_{4}-\widehat{S}_{1} \widehat{S}_{2}}{\widehat{S}_{3}-\widehat{S}_{2}^{2}},
$$

where

$$
\widehat{S}_{1}=\frac{1}{\bar{u}-\underline{u}} \int_{\underline{u}}^{\bar{u}} \Phi^{-1}(\widehat{F}(u)) \mathrm{d} u,
$$




$$
\begin{aligned}
& \widehat{S}_{2}=\frac{1}{\bar{u}-\underline{u}} \int_{\underline{u}}^{\bar{u}} \Phi^{-1}(\widehat{G}(u)) \mathrm{d} u, \\
& \widehat{S}_{3}=\frac{1}{\bar{u}-\underline{u}} \int_{\underline{u}}^{\bar{u}}\left(\Phi^{-1}(\widehat{G}(u))\right)^{2} \mathrm{~d} u,
\end{aligned}
$$

and

$$
\widehat{S}_{4}=\frac{1}{\bar{u}-\underline{u}} \int_{\underline{u}}^{\bar{u}} \Phi^{-1}(\widehat{F}(u)) \Phi^{-1}(\widehat{G}(u)) \mathrm{d} u .
$$

One can represent the above optimization problem and its solution through a different notation. Let $\underline{u}=u_{0}<u_{1}<\cdots<u_{k}=\bar{u}$ be the partition of the interval $[\underline{u}, \bar{u}]$ generated by the observations from the combined sample $\left\{X_{i}\right\} \cup\left\{Y_{i}\right\}$ that lie within $[\underline{u}, \bar{u}]$ (the endpoints $\underline{u}$ and $\bar{u}$ need not be observations). For $i=1, \ldots, k$, define

$$
w_{i}=u_{i}-u_{i-1}, \quad \varphi_{i}=\Phi^{-1}\left(\widehat{F}\left(u_{i-1}\right)\right), \quad \text { and } \quad \psi_{i}=\Phi^{-1}\left(\widehat{G}\left(u_{i-1}\right)\right) .
$$

Since both $\widehat{F}$ and $\widehat{G}$ are piecewise constant on $[u, \bar{u}]$, with possible jumps only at the points $u_{i}$, so are $\Phi^{-1} \circ \widehat{F}$ and $\Phi^{-1} \circ \widehat{G}$, and we may write (4) as

$$
\underset{\mu, \sigma}{\operatorname{minimize}} \sum_{i=1}^{k} w_{i}\left[\varphi_{i}-\left(\mu+\sigma \psi_{i}\right)\right]^{2},
$$

that is, $\widehat{\mu}$ and $\widehat{\sigma}$ are the coefficients of the weighted least-squares simple regression of $\varphi_{1}, \ldots, \varphi_{k}$ against $\psi_{1}, \ldots, \psi_{k}$, with (positive) weights $w_{1}, \ldots, w_{k}$. In this notation, the solution (5) is the wellknown solution of a simple weighted linear regression problem,

$$
\widehat{\mu}=\frac{1}{\bar{u}-\underline{u}} \sum_{1}^{k} w_{i} \varphi_{i}-\widehat{\sigma} \cdot \frac{1}{\bar{u}-\underline{u}} \sum_{1}^{k} w_{i} \psi_{i}
$$

and

$$
\widehat{\sigma}=\frac{\frac{1}{\bar{u}-\underline{u}} \sum_{1}^{k} w_{i} \varphi_{i} \psi_{i}-\left(\frac{1}{\bar{u}-\underline{u}} \sum_{1}^{k} w_{i} \varphi_{i}\right)\left(\frac{1}{\bar{u}-\underline{u}} \sum_{1}^{k} w_{i} \psi_{i}\right)}{\frac{1}{\bar{u}-\underline{u}} \sum_{1}^{k} w_{i} \psi_{i}^{2}-\left(\frac{1}{\bar{u}-\underline{u}} \sum_{1}^{k} w_{i} \psi_{i}\right)^{2}} .
$$

We study the asymptotic properties of these $\widehat{\mu}$ and $\widehat{\sigma}$ in Section 3 .

\section{2 $L_{\infty}$ and $L_{1}$ norm minimization}

Using the notation in (10), solving (3) under the $L_{\infty}$-norm amounts to solving the following least maximum deviation regression problem:

$$
\underset{\mu, \sigma}{\operatorname{minimize}} \max _{i=1, \ldots, k} w_{i}\left|\varphi_{i}-\left(\mu+\sigma \psi_{i}\right)\right| .
$$

Under the $L_{1}$-norm, solving (3) amounts to solving the following least absolute deviation regression problem:

$$
\underset{\mu, \sigma}{\operatorname{minimize}} \sum_{i=1}^{k} w_{i}\left|\varphi_{i}-\left(\mu+\sigma \psi_{i}\right)\right| .
$$

The objective functions of both (13) and (14) are nonlinear in the decision variables $\mu$ and $\sigma$; however, one can recast these problems as linear programs, and solve them easily numerically. We provide the details of these procedures in Appendix B. 


\subsection{Choosing $u$ and $\bar{u}$}

One approach for choosing $u$ and $\bar{u}$, given the remark just preceding Section 2.1, is to set them as far apart as possible given the data, that is, to let $\underline{u}=\max \left\{\min X_{i}, \min Y_{i}\right\}$ and $\bar{u}=\min \left\{\max X_{i}\right.$, max $\left.Y_{i}\right\}$. (Note that in this case either $\Phi^{-1}(\widehat{F}(\bar{u}))=\infty$ or $\Phi^{-1}(\widehat{G}(\bar{u}))=\infty$, but $\bar{u}$ is the only point with this problem, and since its Lebesgue measure is zero, the entire norm is finite, even for $p=\infty$.) While this approach uses as many data points as possible, it also makes the estimation vulnerable to outliers, which may distort the results: the function $\Phi^{-1}$ is not uniformly continuous, and converges rapidly to $-\infty$ or to $\infty$ as its argument $(\widehat{F}(u)$ or $\widehat{G}(u)$, in our setting) tends to 0 or to 1 ; values of $u$ that are close to extreme observations may therefore have too high a leverage. The instability of the $\Phi^{-1}$ transformation applies to all choices of $p$ for the norm, and is acknowledged as a problem by Hsieh and Turnbull (1996), who also used it in the ROC estimation context, though in a different way.

An alternative, remedying approach is to discard a certain fraction of the extreme observations from each end of both populations, and to set $\underline{u}$ and $\bar{u}$ as far apart as the resulting trimmed data permits. More specifically, let $X_{(1)}<X_{(2)}<\cdots<X_{(m)}$ and $Y_{(1)}<Y_{(2)}<\cdots<Y_{(n)}$ be the ordered observations from the healthy and the diseased populations, respectively; given a number $\alpha \in[0,1 / 2)$, we set $\underline{u}=\max \left\{X_{(\alpha m)}, Y_{(\alpha n)}\right\}$ and $\bar{u}=\min \left\{X_{((1-\alpha) m)}, Y_{((1-\alpha) n)}\right\}$. Simulations seem to indicate that setting $\alpha$ around 0.02 yields the best estimates, and we therefore use the value 0.02 in all simulations and data analyses below (see Section 4.2 for more details).

Yet a third approach for choosing $\underline{u}$ and $\bar{u}$ is to assign them fixed, predetermined values, which do not depend on the data. While this approach is hardly practical in the context of small or mid-sized samples, it simplifies the asymptotic analysis, and seems to have very little effect on the distribution of the estimators in large samples. Indeed, simulations show that for large samples, the empirical distribution of the estimators under $\alpha$-trimming is almost indistinguishable from the one obtained under fixed integration endpoints, when these endpoints are the theoretical $\alpha$ and $1-\alpha$ quantiles of the distributions. See Supporting Information Fig. 1 of this article. We follow, then, Hsieh and Turnbull (1996) in their approach for determining the partition boundary points $\left\{\alpha_{i}\right\}$ needed for their method: use fixed values in the asymptotic analysis, and random values, which depend on the data, for studying actual samples (an "adaptive procedure," in their terminology).

\section{Asymptotic Analysis}

In this section we analyze the asymptotics of the minimum-norm estimators $\widehat{\mu}$ and $\widehat{\sigma}$. For simplicity, we assume that the endpoints $\underline{u}$ and $\bar{u}$ are fixed, and that $F(\underline{u}), F(\bar{u}), G(\underline{u}), G(\bar{u}) \in(0,1)$. As usual, we also assume throughout this section that the sample sizes are such that $m=m(n)$, and that $m /(m+n) \rightarrow \lambda \in(0,1)$ as $n \rightarrow \infty$. The proofs are given in Appendix C.

Theorem 3.1 Under the above assumptions, the minimum-norm estimators $\widehat{\mu}$ and $\widehat{\sigma}$ from Eq. (3), with $L_{p}$ norm $(1 \leq p \leq \infty)$, are strongly consistent; that is, $\widehat{\mu} \rightarrow \mu$ and $\widehat{\sigma} \rightarrow \sigma$ almost surely as $n \rightarrow \infty$.

Strong consistency continues to hold also under $\alpha$-trimming of the data, if we add the mild assumption that the sample quantiles of interest converge to the theoretical ones. A sufficient condition for this is that inf $\{u: F(u) \geq \alpha\}$ is the unique solution of $F(u-) \leq \alpha \leq F(u)$, and similarly for $1-\alpha$ and for $G$; see Section 2.3.1 of Serfling (1980).

The asymptotic distribution of $\widehat{\mu}$ and $\widehat{\sigma}$ depends on the choice of $p$ for the $L_{p}$ norm used, and appears to be the most tractable when $p=2$. The result in this case is summarized in the following theorem. 
Theorem 3.2 The asymptotic distribution of the $L_{2}$ estimators $\widehat{\mu}$ and $\widehat{\sigma}$ from Eq. (5), with $\underline{u}$ and $\bar{u}$ fixed, is given by

$$
\sqrt{m(n)+n}\left(\left(\begin{array}{c}
\widehat{\mu} \\
\widehat{\sigma}
\end{array}\right)-\left(\begin{array}{c}
\mu \\
\sigma
\end{array}\right)\right) \Rightarrow N\left(0, V_{\mathbf{S}}^{\prime} \Sigma\left(V_{\mathbf{S}}^{\prime}\right)^{\mathrm{T}}\right) \quad \text { as } n \rightarrow \infty,
$$

where the matrices $V_{\mathbf{S}}^{\prime}$ and $\Sigma$ are defined in the Appendix.

By plugging $\widehat{\mu}$ and $\widehat{\sigma}$ into Eq. (1), we get a natural estimator for the AUC, namely, $\widehat{\mathrm{AUC}}=$ $\Phi\left(\widehat{\mu} / \sqrt{1+\widehat{\sigma}^{2}}\right)$. Theorem 3.1 and the continuous-mapping theorem guarantee that $\widehat{\mathrm{AUC}}$ is a strongly consistent estimate of the AUC. A routine application of the delta method yields that under $L_{2}$ estimation, $\widehat{\mathrm{AUC}}$ is asymptotically normal, with $\sqrt{n}(\widehat{\mathrm{AUC}}-\mathrm{AUC}) \Rightarrow$ $N\left(0, M V_{S}^{\prime} \Sigma\left(V_{S}^{\prime}\right)^{\mathrm{T}} M^{\mathrm{T}}\right)$ as $n \rightarrow \infty$, where

$$
M=\operatorname{\nabla AUC}(\mu, \sigma)=\left(\phi\left(\frac{\mu}{\sqrt{1+\sigma^{2}}}\right),-\phi\left(\frac{\mu}{\sqrt{1+\sigma^{2}}}\right) \frac{\mu \sigma}{\left(1+\sigma^{2}\right)^{3 / 2}}\right) .
$$

\section{Numerical Studies}

\subsection{Estimating $h$}

Even if the ROC curve were known in full detail, one would still need to know $h$ (or at least estimate it) in order to translate sensitivities and specificities from the ROC curve back to the original measurement units of the test, and set corresponding values of $c$. Since our method is based on estimating $h$, this follows naturally. From the equalities in (2), one may estimate $h$ either by $\Phi^{-1} \circ \widehat{F}$ or by $\Phi_{\widehat{\mu}, \widehat{\sigma}}^{-1} \circ \widehat{G}$. One way to combine these two estimators into a single estimator, $\widehat{h}$, is through their weighted mean, with weights corresponding to the sample sizes:

$$
\widehat{h}(u)=\frac{m}{m+n} \Phi^{-1}(\widehat{F}(u))+\frac{n}{m+n}\left(\widehat{\mu}+\widehat{\sigma} \Phi^{-1}(\widehat{G}(u))\right) .
$$

Figure 1 shows the true transformation, $h$, next to an example of the estimated transformation, $\widehat{h}$, for various transformations and sample sizes. Each graph is based on a single realization of the data set. It can be seen that even for the small sample sizes $(m=n=50)$, the estimates are fairly accurate; as expected, the accuracy improves as the sample sizes become larger.

\subsection{Simulation}

We compared the performance of our estimators with that of three other binormal ROC estimators: Metz's Labroc4, (Metz et al., 1998), Cai and Moskowitz's maximum-likelihood estimator (Cai and Moskowitz, 2004), and Cai and Moskowitz's pseudo-maximum-likelihood estimator (ibid). We considered two pairs of sample sizes $(m=n=50$ and $m=n=200)$, three values for the AUC $(0.6$, 0.75 , and 0.9$)$, and two values for $\sigma$ ( 1 and 2$)$; the value of $\mu$ then follows, according to the formula $\mu=\sqrt{1+\sigma^{2}} \Phi^{-1}$ (AUC). Estimates of the bias and the mean-squared error of the estimators, based on 10000 simulated data sets per model, are presented in Tables 1 and 2 . In the minimum-norm methods, the data were trimmed at level $\alpha=0.02$, and $h$ was taken to be the identity transformation (but see below a discussion and results regarding other transformations).

When comparing the three minimum-norm methods among themselves, they seem to perform similarly in terms of bias (except for when AUC $=0.9$ ), each achieving overall the lowest bias in about one-third of the models. In terms of mean-squared error, the $L_{2}$ method achieved almost invariably the best value, though the other two methods were only slightly less efficient, with average relative efficiency of $\sim 95 \%$. 

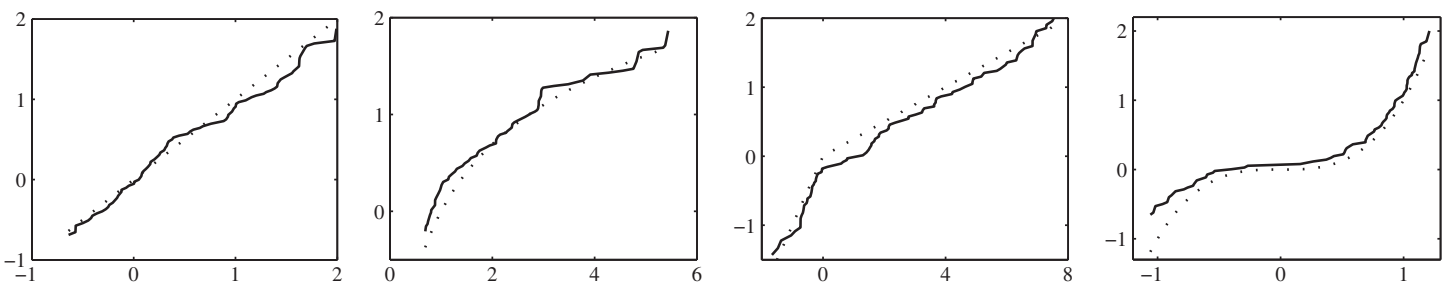

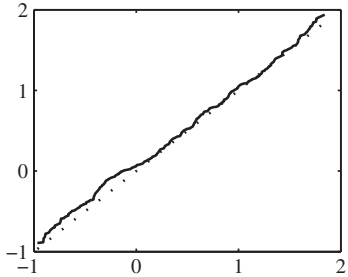

(a)

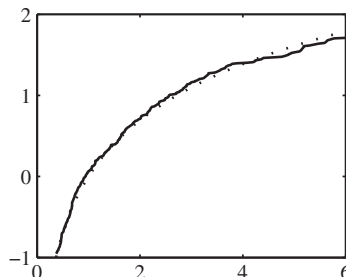

(b)

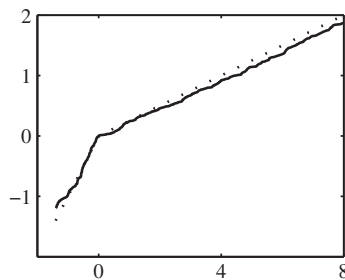

(c)

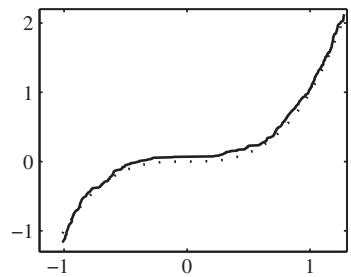

(d)

Figure 1 True transformation $h$ (dotted curves) and estimated transformation $\widehat{h}$ (solid curves), for four transformations: (A) $h(x)=x$; (B) $h(x)=\log (x)$; (C) $h(x)=x 1_{x<0}+\frac{1}{4} x 1_{x \geq 0}$; and (D) $h(x)=x^{3}$. In top panels, $m=n=50$, and in bottom panels, $m=n=200$; in all cases, $\mu=\sigma=1$.

When comparing the $L_{2}$ method with the three methods that are not based on norm minimization, no single method emerges as uniformly better than all the others. It can be said, though, that the $L_{2}$ estimator has achieved the lowest bias in 17 of the 24 cases studied, and that the pseudomaximum-likelihood estimator has achieved the lowest mean-squared error in 16 cases. When comparing the $L_{2}$ and the pseudo-maximum-likelihood estimators, the former achieved an average relative efficiency of $\sim 80 \%$.

Next, we study the effect of varying the trimming level, $\alpha$. Supporting Information Table 1 shows that small values of $\alpha$, around 0.02 , seem to yield the best estimates in terms of the MSE. The bias appears to be too large (in absolute value) for extreme values of $\alpha$ in the range considered, and it varies, yet remains small, between those extremes. Thus, we recommend to trim the data at level 0.02, or at a similar level.

The simulation results do not change considerably when one uses for $h$ transformations other than the identity transformation. Supporting Information Table 2 illustrates this through a small simulation study, in which $h(x)=\log (x)$, and for a few choices of AUC, $\sigma, m$, and $n$ (the numbers should be compared with the appropriate entries in Tables 1 and 2). Other transformations yielded similar results.

All of the simulations above used equal numbers of healthy and diseased subjects, i.e. $m=n$. Supporting Information Fig. 2 shows how the bias and MSE vary as $m$ ranges between 20 and 200 (where $n=400-m$, to keep the total number of subjects constant). While the bias does not appear to be influenced monotonically by $m$, the MSE clearly decreases as the samples become closer in size (i.e. as $m$ increases to 200). Note that the decrease is rather rapid, and already when the ratio between the sample sizes is about 1:3 (corresponding to $m=100$ ), the MSEs are close to their values in the fully symmetric design $(m=200)$.

\subsection{Analysis of clinical data}

Duchenne muscular dystrophy (DMD) is a recessive genetic disorder that causes muscle weakness in male children. Percy et al. (1982) collected data on four biomarkers associated with the disease from 87 normal women (with no DMD family history) and 38 carrier women (mothers or sisters of DMD 
Table 1 The bias, as estimated from simulations, of six methods: the three minimum-norm methods described here $\left(L_{1}, L_{2}\right.$, and $\left.L_{\infty}\right)$; Metz et al.'s Labroc4 (LAB); Cai and Moskowitz's maximum likelihood (MLE); and Cai and Moskowitz's pseudo-maximum-likelihood (PMLE). ${ }^{\text {a) }}$

\begin{tabular}{|c|c|c|c|c|c|c|c|c|c|c|}
\hline \multirow[b]{2}{*}{ AUC } & \multirow[b]{2}{*}{$\sigma$} & \multirow[b]{2}{*}{$m$} & \multirow[b]{2}{*}{$n$} & & \multicolumn{6}{|c|}{ Bias $\times 10^{2}$} \\
\hline & & & & & $L_{1}$ & $L_{2}$ & $L_{\infty}$ & LAB & MLE & PMLE \\
\hline \multirow[t]{8}{*}{0.6} & \multirow[t]{4}{*}{1} & \multirow[t]{2}{*}{50} & \multirow[t]{2}{*}{50} & $\widehat{\mu}$ & -0.494 & -0.847 & -0.134 & 1.481 & 2.100 & 0.210 \\
\hline & & & & $\widehat{\sigma}$ & -1.095 & -0.397 & 1.238 & 1.169 & -3.360 & -0.651 \\
\hline & & \multirow[t]{2}{*}{200} & \multirow[t]{2}{*}{200} & $\widehat{\mu}$ & -0.307 & -0.318 & -0.320 & 0.442 & 0.634 & 0.114 \\
\hline & & & & $\widehat{\sigma}$ & -0.282 & -0.083 & 0.313 & 0.186 & -0.955 & -0.155 \\
\hline & \multirow[t]{4}{*}{2} & \multirow[t]{2}{*}{50} & \multirow[t]{2}{*}{50} & $\widehat{\mu}$ & -1.670 & -1.631 & -1.011 & 3.476 & 4.390 & -2.118 \\
\hline & & & & $\hat{\sigma}$ & -7.585 & -6.996 & -4.859 & 11.385 & -18.239 & -12.044 \\
\hline & & \multirow[t]{2}{*}{200} & \multirow[t]{2}{*}{200} & $\widehat{\mu}$ & -0.286 & -0.448 & -0.269 & 0.633 & 0.969 & -0.696 \\
\hline & & & & $\widehat{\sigma}$ & -2.068 & -2.330 & -1.811 & 2.872 & -8.345 & -3.599 \\
\hline \multirow[t]{8}{*}{0.75} & \multirow[t]{4}{*}{1} & \multirow[t]{2}{*}{50} & \multirow[t]{2}{*}{50} & $\widehat{\mu}$ & -0.892 & -0.887 & 1.342 & 3.577 & 2.389 & 1.256 \\
\hline & & & & $\widehat{\sigma}$ & -0.731 & -0.904 & 1.778 & 1.546 & -2.493 & -0.916 \\
\hline & & \multirow[t]{2}{*}{200} & \multirow[t]{2}{*}{200} & $\widehat{\mu}$ & -0.388 & -0.248 & 0.494 & 0.864 & 0.696 & 0.488 \\
\hline & & & & $\widehat{\sigma}$ & -0.202 & -0.008 & 0.546 & 0.487 & -0.597 & -0.037 \\
\hline & \multirow[t]{4}{*}{2} & \multirow[t]{2}{*}{50} & \multirow[t]{2}{*}{50} & $\widehat{\mu}$ & -2.995 & -3.223 & -0.903 & 8.571 & 1.103 & -7.446 \\
\hline & & & & $\widehat{\sigma}$ & -6.506 & -6.264 & -3.345 & 10.546 & -12.863 & -14.897 \\
\hline & & \multirow[t]{2}{*}{200} & \multirow[t]{2}{*}{200} & $\widehat{\mu}$ & -0.873 & -1.233 & -0.883 & 1.913 & -0.269 & -2.134 \\
\hline & & & & $\widehat{\sigma}$ & -2.420 & -2.492 & -2.112 & 2.807 & -5.219 & -4.293 \\
\hline \multirow[t]{8}{*}{0.9} & \multirow[t]{4}{*}{1} & \multirow[t]{2}{*}{50} & \multirow[t]{2}{*}{50} & $\widehat{\mu}$ & -0.448 & 1.042 & 4.158 & 8.798 & 3.624 & 0.342 \\
\hline & & & & $\widehat{\sigma}$ & -1.675 & 0.039 & 2.962 & 2.852 & -2.513 & -3.432 \\
\hline & & 200 & 200 & $\widehat{\mu}$ & -0.115 & 0.068 & 1.032 & 1.776 & 1.243 & 0.049 \\
\hline & & & & $\widehat{\sigma}$ & -0.423 & -0.002 & 0.700 & 0.625 & -0.242 & -0.829 \\
\hline & 2 & 50 & 50 & $\widehat{\mu}$ & 1.173 & 4.347 & 11.589 & 21.744 & 0.830 & -27.010 \\
\hline & & & & $\widehat{\sigma}$ & 0.553 & 3.355 & 9.147 & 12.071 & -9.902 & -26.580 \\
\hline & & 200 & 200 & $\widehat{\mu}$ & 0.378 & 0.811 & 4.794 & 4.220 & -0.816 & -7.310 \\
\hline & & & & $\widehat{\sigma}$ & 0.487 & 0.929 & 3.537 & 2.487 & -3.254 & -7.566 \\
\hline
\end{tabular}

a) Results are based on 10000 simulated data sets for each method and model.

affected boys). The data set was taken from Andrews and Herzberg (1985), and is available as Supporting Information on this journal's webpage; this very set was used in the context of ROC analysis, for example, by Fluss, Faraggi, and Reiser (2005). For illustration purposes, we focus below on serum levels of creatine kinase $(\mathrm{CK}$, an enzyme that converts creatine to phosphocreatine and adenosine diphosphate) as a single marker, and ignore the other three. To conform with the independence assumption, we omit repeated measurements, keeping only the first one when multiple measurements per woman are available. Table 3 lists the estimates of $\mu, \sigma$, and the AUC for the six methods compared above, and Figure 2 shows the corresponding ROC curves. The six curves are very similar, and in particular, the six AUC estimates are remarkably close to each other (ranging between 0.886 and 0.897 ).

\subsection{Bootstrap}

When computing confidence intervals for $\mu, \sigma$, or other quantities of interest, especially for small or mid-sized samples, the bootstrap method is handy. We studied the coverage probability of $95 \%$ bootstrap confidence intervals, built from empirical quantiles of resampled simulated data sets, for 
Table 2 Estimated mean-squared error of the six methods compared in Table 1.

\begin{tabular}{|c|c|c|c|c|c|c|c|c|c|c|}
\hline \multirow[b]{2}{*}{ AUC } & \multirow[b]{2}{*}{$\sigma$} & \multirow[b]{2}{*}{$m$} & \multirow[b]{2}{*}{$n$} & & \multicolumn{6}{|c|}{ Mean-squared error $\times 10$} \\
\hline & & & & & $L_{1}$ & $L_{2}$ & $L_{\infty}$ & LAB & MLE & PMLE \\
\hline \multirow[t]{8}{*}{0.6} & \multirow[t]{4}{*}{1} & \multirow[t]{2}{*}{50} & \multirow[t]{2}{*}{50} & $\widehat{\mu}$ & 0.423 & 0.423 & 0.453 & 0.465 & 0.427 & 0.434 \\
\hline & & & & $\widehat{\sigma}$ & 0.256 & 0.237 & 0.240 & 0.286 & 0.228 & 0.224 \\
\hline & & \multirow[t]{2}{*}{200} & \multirow[t]{2}{*}{200} & $\widehat{\mu}$ & 0.109 & 0.107 & 0.123 & 0.106 & 0.103 & 0.105 \\
\hline & & & & $\widehat{\sigma}$ & 0.062 & 0.060 & 0.068 & 0.058 & 0.052 & 0.053 \\
\hline & \multirow[t]{4}{*}{2} & \multirow[t]{2}{*}{50} & \multirow[t]{2}{*}{50} & $\widehat{\mu}$ & 1.167 & 1.165 & 1.235 & 1.345 & 1.099 & 1.038 \\
\hline & & & & $\widehat{\sigma}$ & 1.526 & 1.473 & 1.523 & 1.704 & 1.017 & 0.914 \\
\hline & & \multirow[t]{2}{*}{200} & \multirow[t]{2}{*}{200} & $\widehat{\mu}$ & 0.287 & 0.289 & 0.303 & 0.292 & 0.268 & 0.279 \\
\hline & & & & $\widehat{\sigma}$ & 0.351 & 0.328 & 0.354 & 0.302 & 0.283 & 0.261 \\
\hline \multirow[t]{8}{*}{0.75} & \multirow[t]{4}{*}{1} & \multirow[t]{2}{*}{50} & \multirow[t]{2}{*}{50} & $\widehat{\mu}$ & 0.578 & 0.563 & 0.613 & 0.611 & 0.555 & 0.547 \\
\hline & & & & $\widehat{\sigma}$ & 0.394 & 0.365 & 0.398 & 0.346 & 0.289 & 0.264 \\
\hline & & \multirow[t]{2}{*}{200} & \multirow[t]{2}{*}{200} & $\widehat{\mu}$ & 0.140 & 0.136 & 0.147 & 0.127 & 0.130 & 0.132 \\
\hline & & & & $\widehat{\sigma}$ & 0.084 & 0.081 & 0.091 & 0.069 & 0.067 & 0.067 \\
\hline & \multirow[t]{4}{*}{2} & \multirow[t]{2}{*}{50} & \multirow[t]{2}{*}{50} & $\widehat{\mu}$ & 1.871 & 1.757 & 1.908 & 2.047 & 1.528 & 1.332 \\
\hline & & & & $\widehat{\sigma}$ & 1.824 & 1.667 & 1.703 & 2.080 & 1.140 & 1.135 \\
\hline & & \multirow[t]{2}{*}{200} & \multirow[t]{2}{*}{200} & $\widehat{\mu}$ & 0.431 & 0.415 & 0.469 & 0.410 & 0.373 & 0.369 \\
\hline & & & & $\widehat{\sigma}$ & 0.421 & 0.405 & 0.441 & 0.365 & 0.302 & 0.314 \\
\hline \multirow[t]{8}{*}{0.9} & \multirow[t]{4}{*}{1} & \multirow[t]{2}{*}{50} & \multirow[t]{2}{*}{50} & $\widehat{\mu}$ & 1.534 & 1.500 & 1.557 & 1.440 & 1.201 & 0.913 \\
\hline & & & & $\widehat{\sigma}$ & 0.921 & 0.875 & 0.888 & 0.647 & 0.554 & 0.414 \\
\hline & & 200 & 200 & $\widehat{\mu}$ & 0.297 & 0.294 & 0.311 & 0.263 & 0.256 & 0.235 \\
\hline & & & & $\widehat{\sigma}$ & 0.176 & 0.166 & 0.176 & 0.126 & 0.121 & 0.111 \\
\hline & 2 & 50 & 50 & $\widehat{\mu}$ & 6.996 & 7.066 & 7.592 & 6.708 & 4.153 & 2.819 \\
\hline & & & & $\widehat{\sigma}$ & 4.450 & 4.226 & 4.401 & 4.071 & 2.312 & 2.102 \\
\hline & & 200 & 200 & $\widehat{\mu}$ & 1.322 & 1.263 & 1.476 & 0.990 & 0.879 & 0.835 \\
\hline & & & & $\widehat{\sigma}$ & 0.864 & 0.842 & 0.929 & 0.634 & 0.520 & 0.553 \\
\hline
\end{tabular}

various sample sizes and values of the AUC. The results are shown in Table 4, and are indeed close to the target 0.95 probability.

\section{Covariates}

Often, the distributions of $X$ and $Y$ depend on covariates such as age or gender. When the covariates are all categorical, one may partition the data set into a small number of sub-samples (say, according to gender only), and apply any binormal ROC estimation procedure - including the minimum-norm procedure presented above - separately to each sub-sample. This approach is flexible in its modeling ability, as it admits a different $h$ for each covariate level, but inference may become less accurate due to the smaller sample sizes in each application of the procedure. Therefore, regression-type techniques that allow the incorporation of multiple continuous covariates are of great importance.

Alonzo and Pepe (2002) incorporate covariates directly into the shape of the ROC curve, but in accordance with their PDF approach, do not specify how the covariates influence the distribution of the test results. In contrast, Cai and Moskowitz (2004) extend the binormal model to include covariates by assuming (using our notation) that there exists an increasing transformation $h$ such 
Table 3 Estimated parameters for Percy's Duchenne Muscular Disease data.

\begin{tabular}{lllllll}
\hline & $L_{1}$ & $L_{2}$ & $L_{\infty}$ & LAB & MLE & PMLE \\
\hline$\widehat{\mu}$ & 2.137 & 2.236 & 2.537 & 2.331 & 2.242 & 2.272 \\
$\widehat{\sigma}$ & 1.363 & 1.484 & 1.753 & 1.640 & 1.533 & 1.594 \\
$\widehat{A U C}$ & 0.897 & 0.894 & 0.896 & 0.888 & 0.890 & 0.886 \\
\hline
\end{tabular}

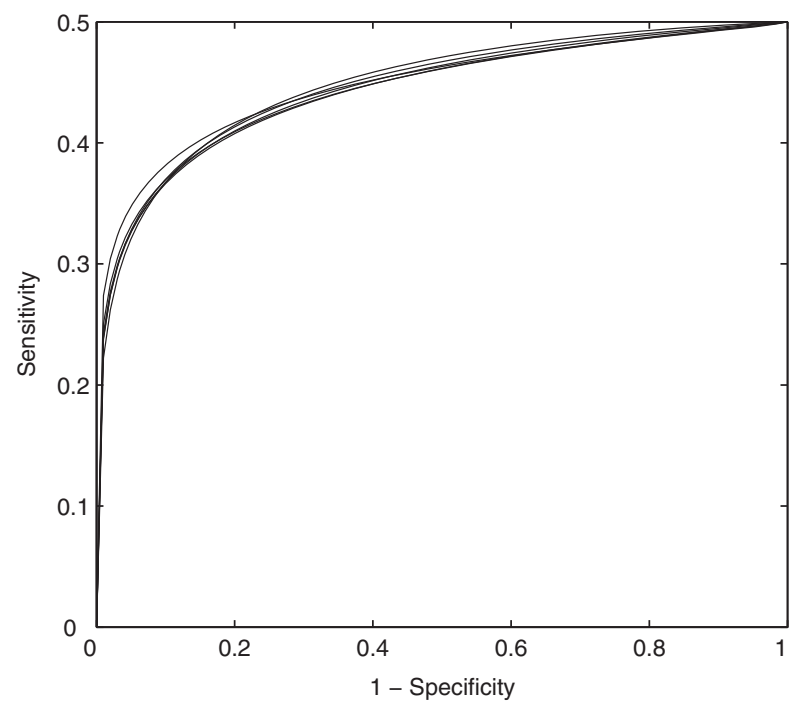

Figure 2 Estimated ROC curves of Percy, Andrews, and Thompson (1982) DMD data, corresponding to the six methods listed is Table 3. The curves are too close to allow a legend indicate which curve corresponds to which method.

that $h(X)-\boldsymbol{\beta}^{\mathrm{T}} \mathbf{Z} \sim N(0,1)$ and $h(Y)-\gamma^{\mathrm{T}} \mathbf{Z} \sim N\left(\mu, \sigma^{2}\right)$, where $Z$ is a covariate vector. One of the two estimation procedures they proposed, the pseudo-maximum-likelihood approach, can be extended to deal with this model.

To incorporate covariates into the norm minimization framework, we assume that there exists an unknown, increasing transformation $h$, such that

$$
h\left(X-\boldsymbol{\beta}^{\mathrm{T}} \mathbf{Z}\right) \sim N(0,1) \text { and } h\left(Y-\gamma^{\mathrm{T}} \mathbf{W}\right) \sim N\left(\mu, \sigma^{2}\right),
$$

where $\mathbf{Z}$ and $\mathbf{W}$ are vectors of covariates corresponding to healthy and diseased subjects, respectively, and $\mu, \sigma, \boldsymbol{\beta}$, and $\boldsymbol{\gamma}$ are parameters to be estimated. The vectors $\mathbf{Z}$ and $\mathbf{W}$ need not have the same length, let alone correspond to the same set of covariates. In the special case where the two vectors do correspond to the same set of covariates, the model in (15) is similar to that of Cai and Moskowitz (2004), but whereas in our model the transformation $h$ acts on the centered values $X-\boldsymbol{\beta}^{\mathrm{T}} \mathbf{Z}$ and $Y-\boldsymbol{\gamma}^{\mathrm{T}} \mathbf{W}$, the centering in Cai and Moskowitz's model takes place after the application of $h$. Although there is no "right model," we feel that our proposal is more realistic, because the covariates act at the scale on which $X$ and $Y$ are measured.

It follows from (15) that the conditional distributions of $X$ and $Y$, given $\mathbf{Z}$ and $\mathbf{W}$, are given by

$$
F_{\mathbf{Z}}(u)=P(X \leq u \mid \mathbf{Z})=\Phi\left(h\left(u-\boldsymbol{\beta}^{\mathrm{T}} \mathbf{Z}\right)\right)
$$


Table 4 Estimated coverage probabilities of $95 \%$ bootstrap confidence intervals for $\mu, \sigma$, and the AUC, using the $L_{2}$ minimum-norm method with $\alpha=0.02$ trimming. ${ }^{\text {a) }}$

\begin{tabular}{|c|c|c|c|c|c|c|}
\hline \multirow{2}{*}{$\begin{array}{l}\text { True AUC } \\
(m, n)\end{array}$} & \multicolumn{2}{|c|}{0.6} & \multicolumn{2}{|c|}{0.75} & \multicolumn{2}{|c|}{0.9} \\
\hline & $(50,50)$ & $(200,200)$ & $(50,50)$ & $(200,200)$ & $(50,50)$ & $(200,200)$ \\
\hline$\mu$ & 0.958 & 0.946 & 0.957 & 0.948 & 0.964 & 0.965 \\
\hline$\sigma$ & 0.951 & 0.957 & 0.958 & 0.970 & 0.972 & 0.976 \\
\hline AUC & 0.955 & 0.944 & 0.960 & 0.957 & 0.956 & 0.953 \\
\hline
\end{tabular}

a) In all cases, $\sigma=1$, and $\mu$ follows from the choice of the true AUC. Results are based on 1000 simulated data sets, each resampled 1000 times.

and

$$
G_{\mathbf{W}}(u)=P(Y \leq u \mid \mathbf{W})=\Phi\left(\frac{h\left(u-\gamma^{\mathrm{T}} \mathbf{W}\right)-\mu}{\sigma}\right) .
$$

Consequently, the ROC curve associated with the covariates $\mathbf{Z}$ and $\mathbf{W}$ can be shown to be

$$
\operatorname{ROC}_{\mathbf{Z}, \mathbf{W}}(t)=1-G_{\mathbf{W}}\left(F_{\mathbf{Z}}^{-1}(1-t)\right)=\Phi\left(\frac{\mu-h\left(h^{-1}\left(\Phi^{-1}(1-t)\right)+\boldsymbol{\beta}^{\mathrm{T}} \mathbf{Z}-\gamma^{\mathrm{T}} \mathbf{W}\right)}{\sigma}\right) .
$$

The parameters $\boldsymbol{\beta}$ and $\gamma$ can be now interpreted as $h$-dependent shape parameters of the ROC curve. Note that when the two covariate vectors correspond to the same set of covariates and $h$ is the identity function, our ROC model coincides with that of Cai and Moskowitz (2004).

We now present two approaches to estimate the model parameters. For the first approach, we note that it follows from (15) that $X \stackrel{d}{=} \boldsymbol{\beta}^{\mathrm{T}} \mathbf{Z}+h^{-1}(N(0,1))$ (here $\stackrel{\text { 'd }}{=}$ ' denotes equality in distribution). Thus, given a sample $\left\{\left(X_{1}, \mathbf{Z}_{1}\right), \ldots,\left(X_{m}, \mathbf{Z}_{m}\right)\right\}$, we may regress $\left\{X_{i}\right\}$ against $\left\{\mathbf{Z}_{i}\right\}$, ignore the resulting estimate of the intercept, and get an estimate $\widehat{\boldsymbol{\beta}}$ for $\boldsymbol{\beta}$. An estimator $\widehat{\boldsymbol{\gamma}}$ for $\boldsymbol{\gamma}$ follows similarly from the sample $\left\{\left(Y_{1}, \mathbf{W}_{1}\right), \ldots,\left(Y_{n}, \mathbf{W}_{n}\right)\right\}$. Next, we note that the distribution of $X-\boldsymbol{\beta}^{\mathrm{T}} \mathbf{Z}$ and $Y-\gamma^{\mathrm{T}} \mathbf{W}$ is the same as that of $X$ and $Y$ in the original, covariate-free model, with $F_{0}$ and $G_{0}$ replacing $F$ and $G$. Thus, for fixed $\boldsymbol{\beta}$ and $\gamma$, we define

$$
\widehat{F}_{\boldsymbol{\beta}}(u)=\frac{1}{m} \sum_{i=1}^{m} I_{\left\{X_{i}-\boldsymbol{\beta}^{\mathrm{T}} \mathbf{Z}_{i} \leq u\right\}} \quad \text { and } \quad \widehat{G}_{\gamma}(u)=\frac{1}{n} \sum_{i=1}^{n} I_{\left\{Y_{i}-\gamma^{\mathrm{T}} \mathbf{W}_{i} \leq u\right\}} .
$$

Minimum-norm estimates of $\mu$ and $\sigma$ can now be obtained from Eq. (3), with $\widehat{F}_{\widehat{\boldsymbol{\beta}}}$ and $\widehat{G}_{\widehat{\gamma}}$ replacing $\widehat{F}$ and $\widehat{G}$. An estimate of $h$ follows just as in Section 4.1.

An alternative approach is to estimate simultaneously all the model parameters. Using the same line of reason as the one leading to Eq. (3), we define

$$
(\widehat{\mu}, \widehat{\sigma}, \widehat{\boldsymbol{\beta}}, \widehat{\gamma})=\underset{\mu, \sigma, \boldsymbol{\beta}, \gamma}{\arg \min }\left\|\Phi^{-1} \circ \widehat{F}_{\boldsymbol{\beta}}-\left(\mu+\sigma \Phi^{-1} \circ \widehat{G}_{\gamma}\right)\right\| .
$$

This latter approach may be applied to a more general ROC covariate model. One can replace (15) with the assumption that $h\left(R_{X}(X, \mathbf{Z} ; \boldsymbol{\beta})\right) \sim N(0,1)$ and $h\left(R_{Y}(Y, \mathbf{W} ; \gamma)\right) \sim N\left(\mu, \sigma^{2}\right)$, for two functions $R_{X}(\cdot, \cdot ; \boldsymbol{\beta})$ and $R_{Y}(\cdot, \cdot ; \boldsymbol{\gamma})$ known up to the parameter vectors $\boldsymbol{\beta}$ and $\boldsymbol{\gamma}$ (the previous special case is $R_{X}(X, \mathbf{Z} ; \boldsymbol{\beta})=X-\boldsymbol{\beta}^{\mathrm{T}} \mathbf{Z}$, and $\left.R_{Y}(Y, \mathbf{W} ; \boldsymbol{\gamma})=Y-\boldsymbol{\gamma}^{\mathrm{T}} \mathbf{W}\right)$. We then define

$$
\widehat{F}_{\boldsymbol{\beta}}(u)=\frac{1}{m} \sum_{i=1}^{m} I_{\left\{R_{X}\left(X_{i}, \mathbf{Z}_{i} ; \boldsymbol{\beta}\right) \leq u\right\}} \quad \text { and } \quad \widehat{G}_{\gamma}(u)=\frac{1}{n} \sum_{i=1}^{n} I_{\left\{R_{Y}\left(Y_{i}, \mathbf{W}_{i} ; \gamma\right) \leq u\right\}},
$$

and obtain the estimators through (16). However, even under the relatively simple covariate model (15), and regardless of the choice of norm, the minimization problem in (16) can no longer be 
reduced to a regression problem, and is not convex. We therefore do not pursue this approach further.

To illustrate how the first estimation approach may be used, we conducted a small simulation study, with unidimensional covariates and parameters $\mu=0.9539$ and $\sigma=1$ (corresponding to $\mathrm{AUC}=0.75), \boldsymbol{\beta}=0.5, \gamma=1.5, h(x)=x$, and $\mathbf{Z}_{i}, \mathbf{W}_{i} \sim U(0,1)$; the norm used was $L_{2}$ with 0.02 -trimming. The results are summarized in Table 5. It is not possible to compare our estimators with those of other procedures, as the covariate model underlying our approach is different from those underlying all other approaches. When the sample sizes are small, and the transformation $h$ is changed to a nonlinear one, the performance of the estimators for $\mu$ and $\sigma$ appears to decline; more research is needed to address this problem.

\section{Discussion}

We have presented in this work a new estimation approach for binormal ROC curves, which is based on norm minimization. Of the three norms we considered $-L_{1}, L_{2}$, and $L_{\infty}$ - we recommend using the $L_{2}$ estimator, as it was found in simulation to perform slightly better, in addition to being the most tractable mathematically.

Although motivated differently, our method may be thought of as supplementing the work of Hsieh and Turnbull (1996). Roughly speaking, Hsieh and Turnbull's method for grouped data is to estimate $\mu$ and $\sigma$ through the regression of $\Phi^{-1}\left(\widehat{F}\left(\widehat{G}\left({ }^{-1}\left(\alpha_{i}\right)\right)\right)\right)$ against $\Phi^{-1}\left(\alpha_{i}\right)$ for certain exogenously chosen $\alpha_{i} \in[0,1]$; as the authors acknowledge, grouping of the data (which is induced by the choice of the $\alpha_{i}$ ) results in some loss in efficiency. In the last section of their article, Hsieh and Turnbull suggest to estimate $\mu$ and $\sigma$ to be the values that minimize the $L_{2}$ distance between the empirical and the theoretical ordinal dominance curves, that is, that minimize $\int_{0}^{1}\left[\widehat{F}\left(\widehat{G}^{-1}(t)\right)-\Phi\left(\mu+\sigma \Phi^{-1}(t)\right)\right]^{2} \mathrm{~d} t$. This minimization problem is the continuous counterpart of their previous, discrete regression problem (up to a $\Phi^{-1}$ transformation), yet it appears to be less tractable, and is left open (only the asymptotic properties of the estimators are derived, through the theory developed by Millar, 1984). We circumvent the minimization difficulties by restoring the $\Phi^{-1}$ transformation and using the change of variables $u=\widehat{G}(t)$, which produce a variant of the problem that enjoys a simple, closed-form solution, given in Eqs. (11) and (12). Although this solution seems similar to Hsieh and Turnbull's grouped data solution, the two are different both conceptually (there is no data grouping in our solution), and in the details (we solve a weighted regression of $\Phi^{-1}\left(\widehat{F}\left(\left(u_{i}\right)\right)\right)$ against $\Phi^{-1}\left(\widehat{G}\left(u_{i}\right)\right)$, where the weights and the $u_{i} \in \mathbb{R}$ are dictated by the data).

As mentioned in Section 4.1, our approach provides an immediate estimator for the unknown transformation $h$, which is necessary for setting the value of $c$ once sensitivity and specificity are chosen on the ROC curve. This estimator is based on the entire data (both healthy and diseased samples), in contrast with the empirical estimators of $h$ in the PDF approach, which are based only on the healthy sample.

Unlike Metz et al.'s Labroc (Metz et al., 1998), the two methods proposed by Cai and Moskowitz (2004), and the method of Zhou and Lin (2008), our closed-form $L_{2}$ estimator does not require iterative procedures, and is thus free of convergence problems. The approaches of Pepe

Table 5 Estimated bias and mean-squared error (in parentheses) of the estimators under the covariate model (15). ${ }^{\mathrm{a})}$

\begin{tabular}{lllllllll}
\hline & \multicolumn{2}{c}{$\mu$} & \multicolumn{2}{c}{$\sigma$} & \multicolumn{2}{c}{$\boldsymbol{\beta}$} & \multicolumn{2}{c}{$\gamma$} \\
\hline$m, n=50$ & 0.019 & $(0.321)$ & -0.001 & $(0.100)$ & -0.010 & $(0.257)$ & 0.000 & $(0.245)$ \\
$m, n=200$ & 0.001 & $(0.062)$ & -0.001 & $(0.017)$ & -0.002 & $(0.060)$ & 0.005 & $(0.060)$
\end{tabular}

a) Results are based on 10000 simulated data sets for each of the two models. 
(2000) and Alonzo and Pepe (2002) are also implicitly iterative, as they are reduced to solving a generalized linear model. As a result, the code for our estimators runs extremely fast - a fraction of a second on a standard PC, even for a problem of size $m=n=1000$. The code (in Matlab) is available as Supporting Information on this journal's webpage.

To estimate the variance of our estimators (e.g. for computing confidence intervals), one could use, in principle, either the bootstrap approach discussed in Section 4.4, or the appropriate asymptotic expressions from Section 3, with $\widehat{F}$ and $\widehat{G}$ plugged in for $F$ and $G$, and $m /(m+n)$ plugged in for $\lambda$. We recommend the bootstrap approach, as it is simpler to implement, more stable numerically (does not involve numerical integration), and is not geared only toward large samples. In addition, since our estimators are very fast to compute, a bootstrap confidence interval can be computed in a few seconds.

Our estimator for $h$, the function $\widehat{h}$ from Section 4.1, may be used as a diagnostic tool, to test the validity of the binormality assumption. We pursue this idea in a sequel to this study.

Acknowledgements We thank the Associate Editor and two anonymous referees for their constructive comments.

\section{Conflict of Interests Statement}

The authors have declared no conflict of interest.

\section{Appendix A}

Lemma A.1 Let $W$ be a continuous random variable, and $h_{1}, h_{2}: \mathbb{R} \rightarrow \mathbb{R}$ be two continuous functions that are strictly increasing on the support of $W$. If $h_{1}(W)$ and $h_{2}(W)$ have the same law, then $h_{1}=h_{2}$ on the support of $W$.

Proof. Suppose by contradiction that there exists $w_{0} \in \operatorname{supp}(W)$ such that $h_{1}\left(w_{0}\right)<h_{2}\left(w_{0}\right)$, say, and let $\gamma=h_{1}\left(w_{0}\right)$. Since $h_{1}$ is continuous, $w_{0}$ may be taken to belong, without loss of generality, to the interior of $\operatorname{supp}(W)$, so that there exists a neighborhood $N$ of $w_{0}$ such that $P(W<w)$ is positive and strictly increasing in $w$ on $N$.

Now distinguish between two cases: If the infimum of the image of $h_{2}$ is greater than $\gamma$, then $P\left(h_{2}(W) \leq \gamma\right)=0$, but by the monotonicity of $h_{1}$, we get that $P\left(h_{1}(W) \leq \gamma\right)=P\left(W \leq w_{0}\right)>0$, which is a contradiction to the assumption that $h_{1}(W)$ and $h_{2}(W)$ have the same law. Otherwise, by the continuity of $h_{2}$, there exists a point $w^{\prime}$ such that $h_{2}\left(w^{\prime}\right)=\gamma$. It then follows from the monotonicity of $h_{2}$ and from the assumption $h_{1}\left(w_{0}\right)<h_{2}\left(w_{0}\right)$ that $w^{\prime}<w_{0}$, whence $P\left(h_{2}(W) \leq \gamma\right)=P\left(W \leq w^{\prime}\right)<P\left(W \leq w_{0}\right)=P\left(h_{1}(W) \leq \gamma\right)$. This contradicts again the assumption that $h_{1}(W)$ and $h_{2}(W)$ have the same law, and the proof is complete.

\section{Appendix B}

\section{Linear Programming}

Following Wagner (1959), the optimization problem (13) may be formulated as a linear program, after introducing an additional decision variable, $\eta$ :

$$
\begin{aligned}
& \underset{\mu, \sigma, \eta}{\operatorname{minimize} \eta} \\
& \text { subject to }-\eta \leq w_{i}\left[\varphi_{i}-\left(\mu+\sigma \psi_{i}\right)\right] \leq \eta, \quad i=1, \ldots, k .
\end{aligned}
$$


In the standard matrix form of linear programming, this optimization problem becomes

$$
\underset{\boldsymbol{\theta}}{\operatorname{minimize}} \mathbf{c}^{\mathrm{T}} \boldsymbol{\theta} \text { subject to } \mathbf{A} \boldsymbol{\theta} \leq \mathbf{b},
$$

where

$$
\boldsymbol{\theta}=\left(\begin{array}{c}
\mu \\
\sigma \\
\eta
\end{array}\right), \quad \mathbf{c}=\left(\begin{array}{l}
0 \\
0 \\
1
\end{array}\right), \quad \mathbf{A}=\left(\begin{array}{ccc}
-w_{1} & -w_{1} \psi_{1} & -1 \\
-w_{2} & -w_{2} \psi_{2} & -1 \\
& \vdots & \\
-w_{k} & -w_{k} \psi_{k} & -1 \\
w_{1} & w_{1} \psi_{1} & -1 \\
w_{2} & w_{2} \psi_{2} & -1 \\
& \vdots & \\
w_{k} & w_{k} \psi_{k} & -1
\end{array}\right), \quad \mathbf{b}=\left(\begin{array}{c}
-w_{1} \varphi_{1} \\
-w_{2} \varphi_{2} \\
\vdots \\
-w_{k} \varphi_{k} \\
w_{1} \varphi_{1} \\
w_{2} \varphi_{2} \\
\vdots \\
w_{k} \varphi_{k}
\end{array}\right)
$$

Similarly, (14) can be also linearized, this time by introducing $k$ new decision variables, $\eta_{1}, \ldots, \eta_{k}$, as follows:

$$
\begin{aligned}
& \underset{\mu, \sigma, \eta_{i}}{\operatorname{minimize}} \sum_{i=1}^{k} \eta_{i} \\
& \text { subject to }-\eta_{i} \leq w_{i}\left[\varphi_{i}-\left(\mu+\sigma \psi_{i}\right)\right] \leq \eta_{i}, \quad i=1, \ldots, k .
\end{aligned}
$$

In the standard matrix notation of linear programming, the problem is identical to (B1), but this time with

$$
\boldsymbol{\theta}=\left(\begin{array}{c}
\mu \\
\sigma \\
\eta_{1} \\
\vdots \\
\eta_{k}
\end{array}\right), \quad \mathbf{c}=\left(\begin{array}{c}
0 \\
0 \\
1 \\
\vdots \\
1
\end{array}\right), \quad \mathbf{b}=\left(\begin{array}{c}
-w_{1} \varphi_{1} \\
-w_{2} \varphi_{2} \\
\vdots \\
-w_{k} \varphi_{k} \\
w_{1} \varphi_{1} \\
w_{2} \varphi_{2} \\
\vdots \\
w_{k} \varphi_{k}
\end{array}\right), \quad \mathbf{A}=\left(\begin{array}{cccccc}
-w_{1} & -w_{1} \psi_{1} & -1 & & & \\
-w_{2} & -w_{2} \psi_{2} & & -1 & & \\
& \vdots & & & \ddots & \\
-w_{k} & -w_{k} \psi_{k} & & & & -1 \\
w_{1} & w_{1} \psi_{1} & -1 & & & \\
w_{2} & w_{2} \psi_{2} & & -1 & & \\
& \vdots & & & \ddots & \\
w_{k} & w_{k} \psi_{k} & & & & -1
\end{array}\right) .
$$

\section{Appendix C}

\section{Consistency and Asymptotic Distribution}

To emphasize the dependence of the analysis on the sample sizes, we denote in the proofs below the empirical distribution functions by $\widehat{F}_{m}$ and $\widehat{G}_{n}$, and the minimum-norm estimators by $\widehat{\mu}_{n}$ and $\widehat{\sigma}_{n}$.

Proof of Theorem 3.1 From the Glivenko-Cantelli Theorem, $\widehat{F}_{m}=\widehat{F}_{m(n)} \rightarrow F$ and $\widehat{G}_{n} \rightarrow G$ uniformly (all limits in this proof are in the almost-sure sense, and as $n \rightarrow \infty$ ). Now define the random functions $\varphi^{n}=\Phi^{-1} \circ \widehat{F}_{m}$ and $\psi^{n}=\Phi^{-1} \circ \widehat{G}_{n}$. From Eq. (2), the equality $\Phi_{\mu, \sigma}^{-1}(x)=\mu+\sigma \Phi^{-1}(x)$, and the assumption that $F(\underline{u}), F(\bar{u}), G(\underline{u}), G(\bar{u}) \in(0,1)$, it follows that both $\varphi^{n}$ and $\mu+\sigma \psi^{n}$ converge uniformly on $[\underline{u}, \bar{u}]$ to the same limit (namely, to the function $h$ ). Thus,

$$
\varphi^{n}-\left(\mu+\sigma \psi^{n}\right) \rightarrow 0
$$


(as a sequence of random functions), and since the last convergence is uniform, we get that $\| \varphi^{n}-$ $\left(\mu+\sigma \psi^{n}\right) \| \rightarrow 0$ (as a sequence of random real numbers), even when $p=\infty$.

By the definition of $\widehat{\mu}_{n}$ and $\widehat{\sigma}_{n}$ (Eq. 3), we have that $\left\|\varphi^{n}-\left(\widehat{\mu}_{n}+\widehat{\sigma}_{n} \psi^{n}\right)\right\| \leq\left\|\varphi^{n}-\left(\mu+\sigma \psi^{n}\right)\right\|$ for all $n$, so that $\left\|\varphi^{n}-\left(\widehat{\mu}_{n}+\widehat{\sigma}_{n} \psi^{n}\right)\right\| \rightarrow 0$, and hence $\varphi^{n}-\left(\widehat{\mu}_{n}+\widehat{\sigma}_{n} \psi^{n}\right) \rightarrow 0$. Subtracting (C1) from this last convergence result, we get that

$$
\left(\widehat{\mu}_{n}-\mu\right)+\left(\widehat{\sigma}_{n}-\sigma\right) \psi^{n} \rightarrow 0 .
$$

As noted right before (C1), we have that $\mu+\sigma \psi^{n} \rightarrow h$ on $[\underline{u}, \bar{u}]$, so that $\psi^{n} \rightarrow(h-\mu) / \sigma$. The function $(h-\mu) / \sigma$ is increasing and, in particular, non-constant, and therefore the convergence in (C2) can take place only if $\widehat{\mu}_{n}-\mu \rightarrow 0$ and $\widehat{\sigma}_{n}-\sigma \rightarrow 0$ or, equivalently, only if $\widehat{\mu}_{n} \rightarrow \mu$ and $\widehat{\sigma}_{n} \rightarrow \sigma$. (If $\psi^{n}$ converged to a constant function, say to the constant $r$, then the convergence in (C2) could have taken place also if, for instance, $\widehat{\mu}_{n}-\mu \rightarrow-r$, and $\widehat{\sigma}_{n}-\sigma \rightarrow 1$.) This completes the proof.

Proof of Theorem 3.2 In this proof, $\mathbf{S}=\left(S_{1}, \ldots, S_{4}\right)^{\mathrm{T}}$ denotes the non-random expressions corresponding to $\widehat{\mathbf{S}}=\left(\widehat{S}_{1}, \ldots, \widehat{S}_{4}\right)^{\mathrm{T}}$ from Eqs. (6)-(9), in which $F$ and $G$ replace $\widehat{F}_{m}$ and $\widehat{G}_{n}$ (i.e. $S_{1}=(\bar{u}-\underline{u})^{-1} \int_{\underline{u}}^{\bar{u}} \Phi^{-1}(F(u)) \mathrm{d} u$, etc. $)$.

We first derive the asymptotic joint distribution of $\widehat{S}_{1}, \ldots, \widehat{S}_{4}$. The following analysis is based on the functional delta method as described by van der Vaart (1998).

The random integral $\widehat{S}_{1}$ may be viewed as a functional, say $T(\cdot)$, of $\widehat{F}_{m}$. The first-order Taylor expansion of $T\left(\widehat{F}_{m}\right)$ around $F$ gives

$$
T\left(\widehat{F}_{m}\right)-T(F)=T_{F}^{\prime}\left(\widehat{F}_{m}-F\right)+o\left(m^{-1 / 2}\right),
$$

where the derivative of $T$ at the point $F$ in the "direction" $H$ is

$$
T_{F}^{\prime}(H)=\left.\frac{\mathrm{d}}{\mathrm{d} t} T(F+t H)\right|_{t=0}=\frac{1}{\bar{u}-\underline{u}} \int_{\underline{u}}^{\bar{u}} \frac{H(u)}{\phi\left(\Phi^{-1}(F(u))\right)} \mathrm{d} u .
$$

(Here $\phi$ is the density function of the standard normal distribution.)

From the linearity of $T_{F}^{\prime}(H)$ in $H$, and from the equality $\widehat{F}_{m}(u)=m^{-1} \sum \mathbf{1}_{\left\{X_{i} \leq u\right\}}$, it follows that

$$
T\left(\widehat{F}_{m}\right)-T(F)=\frac{1}{m} \sum_{i=1}^{m} \frac{1}{\bar{u}-\underline{u}} \int_{\underline{u}}^{\bar{u}} \frac{\mathbf{1}_{\left\{X_{i} \leq u\right\}}-F(u)}{\phi\left(\Phi^{-1}(F(u))\right)} \mathrm{d} u+o\left(m^{-1 / 2}\right),
$$

so the difference $T\left(\widehat{F}_{m}\right)-T(F)$ behaves asymptotically like an average of $m$ i.i.d. random variables, each having zero mean and variance

$$
\sigma_{1}^{2}:=\int_{-\infty}^{\infty}\left[\frac{1}{\bar{u}-\underline{u}} \int_{\underline{u}}^{\bar{u}} \frac{\mathbf{1}_{\{x \leq u\}}-F(u)}{\phi\left(\Phi^{-1}(F(u))\right)} \mathrm{d} u\right]^{2} \mathrm{~d} F(x) .
$$

Therefore, $\sqrt{m}\left(\widehat{S}_{1}-S_{1}\right) \Rightarrow N\left(0, \sigma_{1}^{2}\right)$ as $m \rightarrow \infty$. When scaling by a $\sqrt{m+n}$ factor and letting $n$ be the index that approaches infinity (recall that $m=m(n)$, and that $m /(m+n) \rightarrow \lambda$ ), we get that $\sqrt{m+n}\left(\widehat{S}_{1}-S_{1}\right) \Rightarrow N\left(0, \sigma_{1}^{2} / \lambda\right)$ as $n \rightarrow \infty$.

After repeating the above analysis for the remaining $\widehat{S}_{i}$ 's and for their pairwise products, we get that $\sqrt{m+n}(\widehat{\mathbf{S}}-\mathbf{S}) \Rightarrow N(0, \Sigma)$ as $n \rightarrow \infty$, where the non-zero elements of the $4 \times 4$ covariance 
matrix $\Sigma$ are

$$
\begin{aligned}
& \Sigma_{22}=\frac{1}{1-\lambda} \int_{-\infty}^{\infty}\left[\frac{1}{\bar{u}-\underline{u}} \int_{\underline{u}}^{\bar{u}} \frac{\mathbf{1}_{\{u \leq y\}}-G(u)}{\phi\left(\Phi^{-1}(G(u))\right)} \mathrm{d} u\right]^{2} \mathrm{~d} G(y), \\
& \Sigma_{33}=\frac{1}{1-\lambda} \int_{-\infty}^{\infty}\left[\frac{1}{\bar{u}-\underline{u}} \int_{\underline{u}}^{\bar{u}} \frac{2 \Phi^{-1}(G(u))\left[\mathbf{1}_{\{u \leq y\}}-G(u)\right]}{\phi\left(\Phi^{-1}(G(u))\right)} \mathrm{d} u\right]^{2} \mathrm{~d} G(y), \\
& \Sigma_{44}=\frac{1}{\lambda} \int_{-\infty}^{\infty}\left[\frac{1}{\bar{u}-\underline{u}} \int_{\underline{u}}^{\bar{u}} \frac{\Phi^{-1}(F(u))\left[\mathbf{1}_{\{u \leq y\}}-G(u)\right]}{\phi\left(\Phi^{-1}(G(u))\right)} \mathrm{d} u\right]^{2} \mathrm{~d} G(y), \\
& +\frac{1}{1-\lambda} \int_{-\infty}^{\infty}\left[\frac{1}{\bar{u}-\underline{u}} \int_{\underline{u}}^{\bar{u}} \frac{\Phi^{-1}(G(u))\left[\mathbf{1}_{\{u \leq x\}}-F(u)\right]}{\phi\left(\Phi^{-1}(F(u))\right)} \mathrm{d} u\right]^{2} \mathrm{~d} F(x), \\
& \Sigma_{14}=\Sigma_{41}=\frac{1}{\lambda(\bar{u}-\underline{u})^{2}} \\
& \times \int_{-\infty}^{\infty}\left[\int_{\underline{u}}^{\bar{u}} \frac{\mathbf{1}_{\{u \leq x\}}-F(u)}{\phi\left(\Phi^{-1}(F(u))\right)} \mathrm{d} u \int_{\underline{u}}^{\bar{u}} \frac{\Phi^{-1}(G(u))\left[\mathbf{1}_{\{u \leq x\}}-F(u)\right]}{\phi\left(\Phi^{-1}(F(u))\right)} \mathrm{d} u\right] \mathrm{d} F(x), \\
& \Sigma_{23}=\Sigma_{32}=\frac{1}{(1-\lambda)(\bar{u}-\underline{u})^{2}} \\
& \times \int_{-\infty}^{\infty}\left[\int_{\underline{u}}^{\bar{u}} \frac{\mathbf{1}_{\{u \leq y\}}-G(u)}{\phi\left(\Phi^{-1}(G(u))\right)} \mathrm{d} u \int_{\underline{u}}^{\bar{u}} \frac{2 \Phi^{-1}(G(u))\left[\mathbf{1}_{\{u \leq y\}}-G(u)\right]}{\phi\left(\Phi^{-1}(G(u))\right)} \mathrm{d} u\right] \mathrm{d} G(y), \\
& \Sigma_{24}=\Sigma_{42}=\frac{1}{(1-\lambda)(\bar{u}-\underline{u})^{2}} \\
& \times \int_{-\infty}^{\infty}\left[\int_{\underline{u}}^{\bar{u}} \frac{\mathbf{1}_{\{u \leq y\}}-G(u)}{\phi\left(\Phi^{-1}(G(u))\right)} \mathrm{d} u \int_{\underline{u}}^{\bar{u}} \frac{\Phi^{-1}(F(u))\left[\mathbf{1}_{\{u \leq y\}}-G(u)\right]}{\phi\left(\Phi^{-1}(G(u))\right)} \mathrm{d} u\right] \mathrm{d} G(y), \\
& \Sigma_{34}=\Sigma_{43}=\frac{1}{(1-\lambda)(\bar{u}-\underline{u})^{2}} \\
& \times \int_{-\infty}^{\infty}\left[\int_{\underline{u}}^{\bar{u}} \frac{2 \Phi^{-1}(G(u))\left[\mathbf{1}_{\{u \leq y\}}-G(u)\right]}{\phi\left(\Phi^{-1}(G(u))\right)} \mathrm{d} u \int_{\underline{u}}^{\bar{u}} \frac{\Phi^{-1}(F(u))\left[\mathbf{1}_{\{u \leq y\}}-G(u)\right]}{\phi\left(\Phi^{-1}(G(u))\right.} \mathrm{d} u\right] \mathrm{d} G(y) .
\end{aligned}
$$

After deriving the asymptotic distribution of $\widehat{S}$, we proceed to derive that of $\widehat{\mu}$ and $\widehat{\sigma}$. By (5), $\widehat{\mu}$ and $\widehat{\sigma}$ are a function $V$ of $\widehat{S}$, where $V: \mathbb{R}^{4} \rightarrow \mathbb{R}^{2}$ is given by

$$
V(w, x, y, z)=\left(\frac{w y-x z}{y-x^{2}}, \frac{z-w x}{y-x^{2}}\right)^{\mathrm{T}} .
$$

This map is (totally) differentiable, and its partial derivative matrix, at the point $(w, x, y, z)^{\mathrm{T}}$, is

$$
V_{(w, x, y, z)}^{\prime}=\left(\begin{array}{cccc}
\frac{y}{y-x^{2}} & \frac{2 w x y-y z-x^{2} z}{\left(y-x^{2}\right)^{2}} & \frac{x z-w x^{2}}{\left(y-x^{2}\right)^{2}} & \frac{-x}{y-x^{2}} \\
\frac{-x}{y-x^{2}} & \frac{2 x z-w x^{2} w y}{\left(y-x^{2}\right)^{2}} & \frac{w x z}{\left(y-x^{2}\right)^{2}} & \frac{1}{y-x^{2}}
\end{array}\right)
$$

From (5), the continuous-mapping theorem, and Theorem 3.1 (applied to the $L_{2}$ norm), we know that $V(\mathbf{S})=(\mu, \sigma)^{\mathrm{T}}$. Using now the finite-dimensional delta method and combining all the above, Theorem 3.2 is proved. 


\section{References}

Alonzo, T. A. and Pepe, M. S. (2002). Distribution-free ROC analysis using binary regression techniques. Biostatistics 3, 421-432.

Andrews, D. F. and Herzberg, A. M. (1985). Data. Springer, New York.

Cai, T. and Moskowitz, C. S. (2004). Semi-parametric estimation of the binormal ROC curve for a continuous diagnostic test. Biostatistics 5, 573-586.

Dorfman, D. D. and Alf Jr. E. (1968). Maximum likelihood estimation of parameters of signal detection theory - a direct solution. Psychometrika 33, 117-124.

Faraggi, D. and Reiser, B. (2002). Estimation of the area under the ROC curve. Statistics in Medicine 21, 3093-3106.

Fluss, R., Faraggi, D., and Reiser, B. (2005). Estimation of the Youden index and its associated cutoff point. Biometrical Journal 47, 458-472.

Gu, J. and Ghosal, S. (2009). Bayesian ROC curve estimation under binormality using a rank likelihood. Journal of Statistical Planning and Inference 139, 2076-2083.

Hanley, J. A. (1996). The use of the binormal model for parametric ROC analysis of quantitative diagnostic tests. Statistics of Medicine 15, 1575-1585.

Hsieh, F. and Turnbull, B. W. (1996). Nonparametric and semiparametric estimation of the receiver operating characteristic curve. The Annals of Statistics 24, 25-40.

Metz, C. E., Herman, B. A. and Shen, J.-H. (1998). Maximum likelihood estimation of receiver operating characteristic (ROC) curves from continuously-distributed data. Statistics in Medicine 17, $1033-1053$.

Millar, P. W. (1984). A general approach to the optimality of minimum distance estimators. Transactions of the American Mathematical Society 286, 377-418.

Ogilvie, J. C., Creelman, C. D. (1968). Maximum likelihood estimation for receiver operating characteristic curve parameters. Journal of Mathematical Psychology 5, 377-391.

Pepe, M. S. (2000). An interpretation of the ROC curve and inference using GLM procedures. Biometrics 56, $352-359$.

Pepe, M. S. (2003). The Statistical Evaluation of Medical Tests for Classification and Prediction. Oxford University Press, Oxford.

Percy, M. E., Andrews, D. F. and Thompson, M. W. (1982). Duchenne muscular dystrophy carrier detection using logistic discrimination: serum creatine kinase, hemopexin, pyruvate kinase, and lactate dehydrogenase in combination. American Journal of Medical Genetics 13, 27-38.

Royden, H. L. (1988). Real Analysis. Prentice-Hall, New Jersey.

Serfling, R. J. (1980). Approximation Theorems of Mathematical Statistics. Wiley, New York.

van der Vaart, A. (1998). Asymptotic Statistics. Cambridge University Press, Cambridge.

Wagner, H. M. (1959). Linear programming techniques for regression analysis. Journal of the American Statistical Association 54, 206-212.

Zhang, Z. and Pepe, M. S. (2005). A linear regression framework for receiver operating characteristic (ROC) curve analysis. Technical report, UW Biostatistics Working Paper Series.

Zhou, X.-H. and Lin, H. (2008). Semi-parametric maximum likelihood estimates for ROC curves of continuous-scale tests. Statistics in Medicine 27, 5271-5290.

Zhou, X.-H., Obuchowski, N. A., McClish, D. K. (2002). Statistical Methods in Diagnostic Medicine. Wiley, New York.

Zou, K. H. and Hall, W. J. (2000). Two transformation models for estimating and ROC curve derived from continuous data. Journal of Applied Statistics 27, 621-633. 